\title{
Interacción warfarina y acetaminofén Evaluación para establecer su relevancia clínica
}

\author{
Warfarin and acetaminophen interaction \\ Assessment to establish its clinical relevance
}

\author{
Mauricio Ceballos, Héctor Holguín, César González, Pedro Amariles \\ - Medellín (Colombia)
}

\section{Resumen}

Objetivo: realizar una revisión estructurada de la interacción warfarina y acetaminofén, buscando establecer su relevancia clínica y profundizar en el mecanismo de dicha interacción.

Método: revisión estructurada en PubMed/Medline, de artículos en inglés y español, buscando los términos warfarin AND (acetaminophen OR paracetamol) en el título o resumen. La búsqueda se complementó con las referencias de artículos valorados como importantes. Los trabajos se agruparon en: relacionados con el aumento de sangrado por la interacción warfarina-acetaminofén, o relacionados con el mecanismo de la interacción.

Resultados: se identificaron 45 artículos, de los cuales se incluyeron 15 en la revisión: 11 relacionados con el aumento del riesgo de sangrado por la interacción y cuatro con el mecanismo de la interacción. La gravedad del efecto (aumento de la probabilidad de sangrado) se consideró moderada; mientras que la probabilidad de aparición fue valorada como definida. Además, se identificó una relación entre la dosis de acetaminofén y el riesgo de sangrado. Por su parte, el $\mathrm{N}$-acetil-para-benzoquinona-imina (metabolito del acetaminofén) inhibe enzimas del ciclo de la vitamina $\mathrm{K}$ y tiene un efecto sinérgico con el efecto anticoagulante de la warfarina.

Conclusiones: la relevancia clínica de la interacción warfarina - acetaminofén es de riesgo alto, debido a que la gravedad del efecto (aumento del riesgo de sangrado) es moderada y su probabilidad de presentación es definida. Por tanto, estos dos medicamentos pueden ser utilizados conjuntamente, pero se debe realizar una estricta monitorización. El metabolito $\mathrm{N}$-acetil-para-benzoquinona-imina es el responsable del aumento del efecto anticoagulante de la warfarina. (Acta Med Colomb 2013; 38: 22-27)

Palabras clave: warfarina, acetaminofén, interacción medicamentosa, mecanismo de interacción, evidencia clínica.

\footnotetext{
Abstract

Objective: to make a structured review of the interaction between warfarin and acetaminophen, seeking to establish its clinical relevance and deepen in the mechanism of this interaction.

Method: structured review of PubMed/Medline of articles in English and Spanish, looking warfarin and acetaminophen or paracetamol in the title or abstract. The search was complemented with references of articles rated as important. The papers were grouped in: related to increased bleeding due to warfarin-acetaminophen interaction, or related to the mechanism of the interaction.

Results: we identified 45 articles, of which 15 were included in the review: 11 related to increased risk of bleeding due to the interaction and 4 with the mechanism of the interaction. The severity of the effect (increased likelihood of bleeding) was considered moderate, whereas the probability of appearance was rated as definite. In addition, we identified a relationship between the dose of acetaminophen and the risk of bleeding. In turn, the $\mathrm{N}$-acetyl-para-benzoquinone-imine (metabolite of acetaminophen) inhibits enzymes of the vitamin $\mathrm{K}$ cycle and has a synergistic effect with the anticoagulant effect of warfarin.

Conclusions: the clinical relevance of the interaction warfarin-acetaminophen is of high risk due to the fact that the severity of the effect (increased risk of bleeding) is moderate and the probability
}

Dr. Mauricio Ceballos: MSc Epidemiología, Facultad de Medicina, Universidad CES. Grupo de Investigación, Promoción y Prevención Farmacéutica, Universidad de Antioquia; Dr. Héctor Holguín: Químico Farmacéutico, Facultad de Química Farmacéutica, Universidad de Antioquia. Grupo de Investigación, Promoción y Prevención Farmacéutica, Universidad de Antioquia; Dr. César González: MSc Epidemiología, Facultad de Medicina, Universidad CES, Medellín; Dr. Pedro Amariles: Doctor en Farmacia, Facultad de Química Farmacéutica, Universidad de Antioquia. Grupo de Investigación, Promoción y Prevención Farmacéutica, Universidad de Antioquia. Medellín (Colombia).

Correspondencia. Prof. Pedro Amariles. Departamento de Farmacia. Universidad de Antioquia. Medellín (Colombia).

E-mail: pamaris@farmacia.udea.edu.co Recibido: 26/VII/2012 Aceptado: 25/II/2013 
of its presentation is definite. Therefore, these two drugs can be used together, but a strict monitoring should be conducted. The metabolite $\mathrm{N}$-acetyl-para-benzoquinone-imine is responsible for the increase in the anticoagulant effect of warfarin. (Acta Med Colomb 2013; 38: 22-27)

Keywords: warfarin, acetaminophen, drug interaction, interaction mechanism, clinical evidence.

\section{Introducción}

La warfarina es un anticoagulante oral ampliamente utilizado con el objetivo de reducir los problemas tromboembólicos en pacientes con fibrilación atrial, trombosis venosa profunda, tromboembolismo pulmonar o con implantación de prótesis en las válvulas cardiacas (1). Además, es uno de los medicamentos que causa más ingresos hospitalarios, situación que se atribuye, además de su reconocida variabilidad farmacocinética y farmacodinámica $(2,3)$, la variabilidad genética de la isoenzima CYP2C9 (4) y a su utilización en pacientes de edad avanzada (5), a la alta probabilidad que tiene de presentar interacciones medicamentosas.

En la farmacoterapia, las interacciones medicamentosas son un tema importante, debido a que su presentación puede causar problemas de efectividad o seguridad de la farmacoterapia. Por ello, el conocimiento del mecanismo de la interacción y su efecto sobre la efectividad o seguridad de la farmacoterapia facilita a los profesionales de la salud definir la estrategia farmacoterapéutica con mayor probabilidad de efectividad y seguridad (6). En el caso de los pacientes en tratamiento crónico con warfarina puede ser necesaria la utilización de analgésicos, los cuales pueden aumentar el riesgo de hemorragia. En este sentido, existe evidencia de la capacidad que tienen los analgésicos antiinflamatorios no esteroideos (AINEs), por diferentes mecanismos, de presentar interacción con la warfarina (7). Sin embargo, en el caso del acetaminofén (o paracetamol), existe controversia de la evidencia disponible sobre el efecto y, por tanto, la relevancia clínica de dicha interacción. En este contexto, el objetivo de este trabajo fue realizar una revisión estructurada sobre la interacción warfarina y acetaminofén, buscando establecer su relevancia clínica y profundizar en el mecanismo de dicha interacción.

\section{Método}

Revisión estructurada en PubMed/Medline de trabajos relacionados con la interacción warfarina - acetamionofén. Para ello, las palabas warfarin AND (acetaminophen OR paracetamol) en el título o el resumen fueron utilizadas como estrategia de búsqueda. Los criterios de inclusión fueron: artículos en inglés o español, realizados en humanos y publicados entre enero de 1997 y marzo de 2011 y con acceso a texto completo. Los autores revisaron los artículos, incluyendo los que presentaban resultados relacionados directamente con el objetivo de la revisión, los cuales se agruparon en dos categorías, acordes con el aumento de sangrado por la interacción warfarina - acetaminofén, o con el mecanismo de la interacción. Luego de la lectura crítica, se realizó un consenso uno a uno los estudios y, en caso de ser necesario, para resolver discrepancias, se utilizaron herramientas de la evaluación crítica de revisiones. Además, la revisión se complementó con las referencias de artículos considerados como relevantes

\section{Resultados}

La estrategia de búsqueda identificó 45 artículos, de los cuales se incluyeron 15 trabajos con una clara relación con el objetivo de la revisión. De ellos, 11 trabajos relacionados con el efecto de la interacción warfarina - acetaminofén, los cuales mostraron una relación directa entre la dosis de acetaminofén y el riesgo de sangrado; mientras que cuatro artículos relacionados con el mecanismo de la interacción, y en los cuales se identificó al metabolito N-acetil-parabenzoquinona- imina (NAPQI) como posible causante del aumento de la relación internacional normalizada. En la Figura 1 se presenta el flujo de la inclusión de los artículos en la revisión; y a continuación se detallan conceptos extractados de dichos trabajos.

\section{Aspectos generales}

La warfarina es una mezcla racémica de los enantiómeros, S-warfarina y R-warfarina; alcanza la concentración máxima plasmática a los 90 minutos de su administración, se transporta en la sangre unida a proteínas plasmáticas (especialmente albúmina) en un 99\% (8) y sus dos enantiómeros son metabolizados a nivel hepático por dos vías enzimáticas diferentes, siendo la S-warfarina metabolizada por la isoenzima CYP2C9 y la R-warfarina metabolizada por las isoenzimas CYP1A2 y CYP3A4 (9). En el caso de las interacciones medicamentosas, la inhibición de la S-warfarina (cinco veces más potente que el otro enantiómero), se asocia a una probabilidad de mayor relevancia clínica $(5,10)$.

La warfarina es un antagonista de la vitamina $\mathrm{K}$, mediante la inhibición de la enzima vitamina $K$ epóxido reductasa, la cual se encarga de reducir la vitamina K epóxido (forma inactiva) a vitamina KH2 (forma reducida, activa). La vitamina KH2 activa, por una reacción de carboxilación, los factores de la coagulación II, VII, IX y X, conocidos como factores dependientes de la vitamina $K(5,9)$. La disminución de la vitamina $\mathrm{K}$ implica la reducción de estos factores, presentándose un desequilibrio en la hemostasia y causando la anticoagulación.

La cuantificación o medición de la anticoagulación se realiza con el tiempo de protrombina (TP), factor que res- 


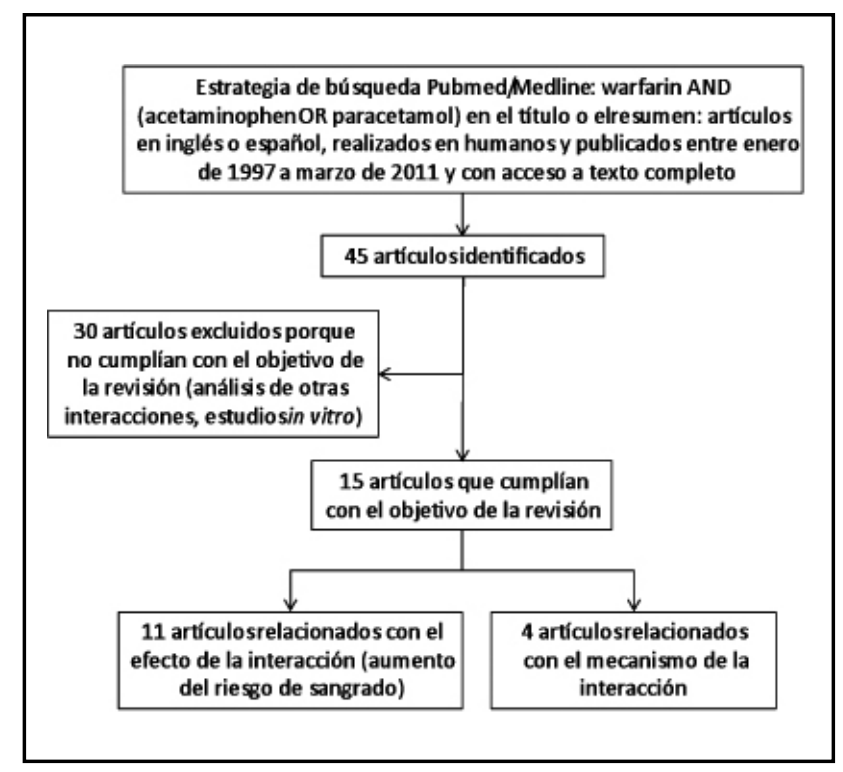

Figura 1. Flujo de la búsqueda, identificación y selección de los trabajos incluidos en la revisión.

ponde a la reducción de los factores II, VII y X. Debido a la variabilidad del TP, se realizó una estandarización de dichos valores, utilizando el concepto de relación internacional normalizada (INR, por sus siglas en inglés) (9) cuyo valor es dependiente del TP del paciente, el TP control y un valor de corrección llamado índice de sensibilidad internacional (ISI). En la mayoría de las indicaciones, el valor del INR debe estar entre 2.0 a $3.0(3,5)$; exceptuando los pacientes con implantación de válvulas cardiacas, donde este valor debe estar entre 2.5 a 3.5 (5).

Los AINEs son ampliamente conocidos por su eficacia para disminuir la inflamación mediada por las prostaglandinas (11). Algunos de ellos interaccionan con la warfarina, debido al efecto que presentan sobre la agregación plaquetaria y/o daño a nivel de la mucosa gástrica. Además, existe evidencia de que algunos de estos fármacos, caso del ácido acetil salicílico, ibuprofeno, naproxeno y celecoxib, desplazan de la warfarina de las proteínas plasmáticas, aumentado en un 3\% la fracción libre, lo cual implica un aumento en tres veces los niveles de warfarina libre y, por tanto, el riesgo de un episodio hemorrágico $(12,13)$.

El acetaminofén es un medicamento preferido para ser utilizado en pacientes en tratamiento anticoagulante que reqiueren de un analgésico-antipirético $(14,15)$, debido a que no tiene efecto sobre la agregación plaquetaria, y no representa un riesgo de lesión de la mucosa gástrica. También, es uno de los más populares antipiréticos y analgésicos de venta libre $(16,17)$. El mecanismo de acción del acetaminofén se basa en la reducción de la síntesis de prostaglandinas, por inhibición de la ciclooxigenasa a nivel del sistema nervioso central $(18,19)$. Con respecto a su farmacocinética, el acetaminofén es absorbido completamente en el intestino delgado, hasta en un $90 \%$ es metabolizado, por glucoronidación o sulfatación, a metabolitos no tóxicos, los cuales se excretan por vía renal. Por su parte, cerca al $10 \%$ del fármaco es metabolizado, por la citocromo P450 (específicamente las isoenzimas CYP2E1, CYP1A2 y CYP3A4), al metabolito intermedio tóxico $\mathrm{N}$-acetil-para-benzoquinona-imina (NAPQI), el cual es glucoronidado y eliminado de forma rápida del organismo (17).

\section{Concepto y tipos de interacción medicamentosa}

Una interacción medicamentosa, corresponde a una modificación, cuantificable, en la magnitud y duración del efecto esperado, provocado bien sea por la administración previa o simultánea de medicamentos (interacciones entre medicamentos), de alimentos (interacciones entre medicamento $\mathrm{y}$ alimento), de productos naturales (interacciones entre medicamento y fitoterapéuticos), así como a condiciones fisiológicas o patológicas propias del paciente (interacciones entre medicamento y enfermedad). Con respecto al mecanismo de la interacción, ésta puede ser farmacocinética, en la cual hay un cambio en la concentración plasmática debido a una modificación en el proceso de absorción, metabolismo, excreción o distribución; o farmacodinámica, cuando hay un cambio en el efecto sin modificación de las concentraciones plasmáticas, asociado a un aumento (sinergismo) o a una disminución del efecto farmacológico (antagonismo) (20).

\section{Efecto de la interacción warfarina y acetaminofén sobre la relación internacional normalizada}

En pacientes en tratamiento con warfarina, la prescripción de acetaminofén se ha identificado como un factor que requiere de un ajuste de la dosis de mantenimiento de la warfarina (21). Situación que ha sido documentada por el reporte de algunos casos, en los cuales, pacientes controlados, al utilizar acetaminofén presentaban un incremento del INR llevando, entre otros problemas de seguridad, a la presentación de un hematoma retroperitoneal (22). En algunos de estos casos, el paciente fue reexpuesto intencionalmente (23) o de forma no intencional (24), lo que ha permitido confirmar la causalidad del acetaminofén en el incremento del INR. Además, algunos estudios muestran que la prescripción conjunta de warfarina y acetaminofén puede ser hasta de un $63 \%(15,25)$. En la Tabla 1 se detallan los aspectos más importantes de los estudios relacionados con el efecto de la interacción warfarina y acetaminofén.

En un estudio de casos y controles (93 casos y 196 controles) realizado por Hylek et al. (3), entre otros, con el objetivo de determinar en un subgrupo de la muestra la asociación de la dosis de acetaminofén con la variación del INR, encontraron una relación dosis dependiente entre la dosis de acetaminofén y la variación del INR. En este sentido, dosis entre 325-2267 mg/semana causó una razón de disparidad (OR por sus siglas en inglés) de 1.1 ([0.5-2.5; IC $95 \%$ ], $\mathrm{p}=0.77$ ); mientras que, con una dosis mayor a 9100 mg/semana, el OR es de 10.0 ([2.6-37.9; IC 95\%], p=0.001). 
Tabla 1. Estudios clínicos controlados o analíticos que evidencian el efecto de la interacción warfarina - acetaminofén.

\begin{tabular}{|c|c|c|c|c|c|c|}
\hline Autor & Diseño del estudio & $\mathbf{N}^{0}$ de pacientes & Dosis de acetaminofén & Exposición (días) & Aumento del INR & Valor $\mathbf{P}$ \\
\hline Hylek et al (3) & $\begin{array}{l}\text { Casos }(52) \text { y } \\
\text { controles (70) }\end{array}$ & $\begin{array}{l}15 \\
11 \\
11 \\
15\end{array}$ & $\begin{array}{c}325-2267 \mathrm{mg} / \mathrm{semana} \\
2275-4549 \mathrm{mg} / \mathrm{semana} \\
4550-9099 \mathrm{mg} / \mathrm{semana} \\
\geq 9100 \mathrm{mg} / \mathrm{semana}\end{array}$ & $\begin{array}{l}\text { Mínimo } 7 \\
\text { Mínimo } 7 \\
\text { Mínimo } 7 \\
\text { Mínimo } 7\end{array}$ & $\begin{array}{l}\text { NR } \\
\text { NR } \\
\text { NR } \\
\text { NR }\end{array}$ & $\begin{array}{c}0.77 \\
0.02 \\
0.001 \\
0.001\end{array}$ \\
\hline Mahé et al (27) & $\mathrm{ECC}$ & 11 & 4000 mg/día & 14 & $\begin{array}{c}1.04 \pm 0.55 \text { vs. } 0.20 \pm 0.32 \\
\text { (con placebo) }\end{array}$ & 0.003 \\
\hline Mahé et al (28) & $\mathrm{ECC}$ & 20 & 4000 mg/día & 14 & $\begin{array}{c}1.20 \pm 0.62 \text { vs } 0.37 \pm 0.48 \\
\text { (con placebo) }\end{array}$ & $<0.001$ \\
\hline Parra et al (29) & ECC & $\begin{array}{l}36 \text { (12 en cada brazo, } \\
\text { incluyendo placebo) }\end{array}$ & $\begin{array}{l}2000 \text { mg/día } \\
4000 \mathrm{mg} \text { /día }\end{array}$ & $\begin{array}{c}14 \\
7 \\
14 \\
21\end{array}$ & $\begin{array}{c}0.7(\text { IC95\% 0.27-1.2) } \\
0.5(\text { IC95\% 0.08-0.9) } \\
0.6(\text { IC95\% 0.21-1) } \\
1.0(\text { IC95\% 0.48-1.5) }\end{array}$ & $\begin{array}{l}0.01 \\
0.04 \\
0.01 \\
0.01\end{array}$ \\
\hline
\end{tabular}

Con el fin de determinar la relevancia clínica de la interacción, también se han realizado algunos estudios prospectivos. En este sentido, Kwan et al (26) realizaron un estudio clínico controlado (ECC) con 20 voluntarios sanos a quienes se les administró $20 \mathrm{mg}$ de warfarina en cuatro situaciones diferentes: (1) warfarina sola, (2) después de tomar acetaminofén 1 g cada cuatro horas durante un día, y (3) después de tomar acetaminofén $1 \mathrm{~g}$ cada cuatro horas durante una semana. Los resultados mostraron que todos los pacientes presentaron una respuesta farmacodinámica a la warfarina igual. Por su parte, Mahé et al. (27), en otro ECC, incluyeron 11 pacientes que tomaban warfarina y tenían un INR estable. Los pacientes fueron asignados aleatoriamente a utilizar acetaminofén 4 $\mathrm{g}$ al día o placebo por 14 días; como resultado se encontró un aumento significativo ( $\mathrm{p}=0.003)$ del INR en los pacientes con acetaminofén, siendo este incremento de $1.04 \pm 0.55$ en la medición del INR comparado con la medición del INR del grupo placebo, la cual fue de $0.20 \pm 0.3$. Los autores también encontraron que el INR aumenta significativamente a partir del cuarto día $(+0.6 \pm 0.6, \mathrm{P}<0.001)$. Posteriormente, estos mismos autores (28), realizaron otro ECC con el objetivo de determinar la variación en el efecto anticoagulante de la warfarina, por la utilización simultánea del acetaminofén, y elucidar el mecanismo de la interacción. En este estudio, 20 pacientes, con un INR estable por 14 días, fueron asignados aleatoriamente a utilizar acetaminofén $1 \mathrm{~g}$ cada seis horas o placebo, y se cuantificó el INR los días 0, 2, 4, 7, 9, 11 y 14. En el grupo que utilizó acetaminofén comparado con el grupo placebo, se encontró un aumento significativo del INR en la primera semana ( $\mathrm{p}=0.0002)$. El aumento máximo de la línea base para los pacientes expuestos fue $1.20 \pm 0.62$ comparado con el grupo control $0.37 \pm 0.48$ ( $\mathrm{p}<0.001)$; a pesar del aumento en el riesgo de sangrado, no se presentó ningún tipo de hemorragia en los pacientes.

Parra et al. (29) realizaron un ECC con 36 pacientes que utilizaban warfarina y con un INR estable, valorado tres semanas antes del estudio. Los pacientes fueron asignados aleatoriamente a utilizar acetaminofén $1 \mathrm{~g}$ dos veces al día y placebo dos veces al día, acetaminofén $1 \mathrm{~g}$ cuatro veces al día, o placebo cuatro veces al día (grupo control).
Los resultados mostraron que a las dos semanas hay una diferencia significativa $(\mathrm{p}=0.01)$ en el INR de los pacientes que utilizaron acetaminofén $2 \mathrm{~g}$ al día y placebo versus los controles. Igual diferencia se encontraba en los pacientes que tomaban $4 \mathrm{~g}$ al día, en las semanas $1(\mathrm{p}=0.04)$, semana $2(\mathrm{p}=0.01)$, y semana $3(\mathrm{p}=0.01)$.

\section{Mecanismo de la interacción}

Algunos trabajos muestran que el aumento del INR en los pacientes anticoagulados con warfarina, debido al uso concomitante de acetaminofén, se debe al aumento de las concentraciones del metabolito tóxico NAPQI del acetaminofén, lo que puede ocurrir por la utilización de dosis altas o por tiempo prolongado del acetaminofén, o por saturación de la glucoronidación en el metabolito. El mecanismo de esta interacción se basa posiblemente en la reacción entre el metabolito tóxico NAPQI y las enzimas del ciclo de la vitamina $K$, interfiriendo al igual que la warfarina, en la producción de los factores de la coagulación dependientes de vitamina $K$, siendo los pacientes de edad avanzada los más sensibles $(28,30,31)$. En esta dirección, en un paciente intoxicado con acetaminofén se identificó un aumento del INR sin el desarrollo de hepatotoxicidad (32). En la Figura 2 se ilustra la forma como se presenta el sinergismo en la inhibición de las enzimas del ciclo de la vitamina $\mathrm{K}$ por parte del metabolito NAPQI, en la terapia anticoagulante con warfarina.

\section{Discusión y recomendaciones \\ Relevancia clínica}

Los resultados de esta revisión estructurada permiten establecer que la utilización simultánea de la warfarina con el acetaminofén puede causar un mayor efecto anticoagulante en el paciente, asociado a un aumento del INR y, por tanto, a un mayor riesgo de sangrado. Sólo un trabajo no presentó alteración en la respuesta por la adición de acetaminofén a la terapia anticoagulante. Sin embargo, la metodología utilizada y las dosis utilizadas de los medicamentos, distantes de las utilizadas en la práctica clínica, limitan sus resultados (26). Por tanto, acorde con la metodología propuesta por 


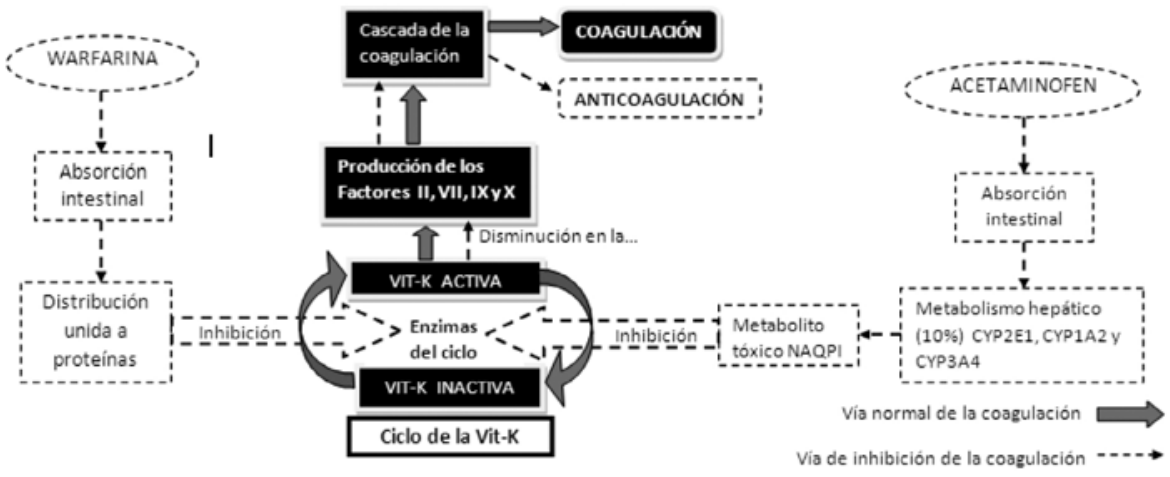

Las flechas oscuras ilustran la via normal de la coagulación, mientras que las line as punte adas indican el mecanismo de acción de la warfarina y el siner gismo presentado por el metabolito tóxico del acetaminofén, mediante la inhibición de las enzimas del ciclo de la vitamina $\mathrm{K}$.

Figura 2. Mecanismo del efecto mediado por la warfarina y el acetaminofén sobre la coagulación.

Amariles et al. (20), la interacción entre la warfarina y el acetaminofén sería de un nivel 2 (riesgo alto). Este nivel se explica por el cruce de la gravedad -moderada- con una probabilidad de aparición -definida-. La valoración de la gravedad de la interacción como Moderada se debe a que, la misma causa una modificación (cambio o interrupción) en la farmacoterapia del paciente. Es importante destacar que, a pesar que la interacción puede causar daño o lesión al paciente por el aumento del INR, en los estudios no se presentaron hemorragias o sangrados que pusieran en riesgo la vida del paciente, excepto en un reporte de caso en el cual un paciente presentó una hemorragia retroperitoneal (22). Por su parte, la valoración de probabilidad de aparición de la interacción como Definida, se debe a que la misma ha sido demostrada mediante ECC.

La evidencia establece que esta interacción se presenta a altas dosis de acetaminofén o por su uso prolongado. En este sentido, el uso de acetaminofén en la terapia anticoagulante con warfarina, según el nivel de relevancia clínica, debe hacerse bajo una estricta monitorización del INR (28); además, para disminuir el riesgo de sangrado, en los casos en los que se requiera, se recomienda utilizar una dosis menor a $2 \mathrm{~g}$ al día por 3-4 días como máximo de acetaminofén $(3,27,29,33)$.

\section{Mecanismo de la interacción}

Los trabajos que abordan este tema muestran que la interacción entre la warfarina y el acetaminofén es de tipo farmacodinámico, debido a que el metabolito tóxico NAQPI del acetaminofén bloquea las enzimas del ciclo de la vitamina $\mathrm{K}$, y al igual que la warfarina, disminuye los factores de la coagulación dependientes de vitamina $\mathrm{K}$, lo que causa un efecto sinérgico en el aumento del INR $(28,30,34)$.

\section{Conflicto de interés}

Los autores certificamos que no tenemos ningún conflicto potencial de interés.

\section{Referencias}

1. Meckley L, Gudgeon J, Anderson J, et al. A policy model to evaluate the benefits, risks and costs of warfarin pharmacogenomic testing. Pharmacoeconomics 2010; 28: 61-74.

2. Fihn S, Callahan C, Martin D, et al. The risk for and severity of bleeding complications in elderly patients treated with warfarin. The National Consortium of Anticoagulation Clinics. Ann Intern Med 1996; 124: 970-9.

3. Hylek E, Heiman H, Skates S, et al. Acetaminophen and other risk factors for excessive warfarin anticoagulation. JAMA 1998; 279: 657-62.

4. Joffe H, Xu R, Johnson F, et al. Warfarin dosing and cytochrome P450 2C9 polymorphisms. Thromb Haemost 2004; 91: 1123-8.

5. Hirsh J, Dalen J, Anderson D, et al. Oral anticoagulants: mechanism of action clinical effectiveness, and optimal therapeutic range. Chest 2001; 119: 8S-21S.

6. Ko Y, Malone D, Skrepnek G, et al. Prescribers' knowledge of and sources of information for potential drug-drug interactions: a postal survey of US prescribers. Drug Saf 2008; 31: 525-36.

7. Yurgaki J, Rodriguez F. Warfarina: uso contemporáneo. Rev Fac Med 2009; 17.

8. Breckenridge A. Oral anticoagulant drugs: pharmacokinetic aspects. Semin Hematol 1978; 15: $19-26$

9. Launiainen T, Sajantila A, Rasanen I, et al. Adverse interaction of warfarin and paracetamol: evidence from a post-mortem study. Eur J Clin Pharmaco. 2010; 66: 97-103.

10. Lehmann D. Enzymatic shunting: resolving the acetaminophen-warfarin controversy. Pharmacotherapy 2000; 20: 1464-8.

11. Su W, Cheng M, Lee W, et al. Nonsteroidal anti-inflammatory drugs for wounds: pain relief or excessive scar formation? Mediators Inflamm 2010: 413238.

12. Christensen H, Baker M, Tucker G, et al. Prediction of plasma protein binding displacement and its implications for quantitative assessment of metabolic drugdrug interactions from in vitro data. J Pharm Sci 2006; 95: 2778-2787.

13. Hersh E, Pinto A, Moore P. Adverse drug interactions involving common prescription and over-the-counter analgesic agents. Clin Ther 2007; 29: 2477-97.

14. Gutthann S, Raiford D. Individual nonsteroidal antiinflammatory drugs and other risk factors for upper gastrointestinal bleeding and perforation. Epidemiology 1997; 8: 18-24.

15. Gasse C, Hollowell J, Meier C, et al. Drug interactions and risk of acute bleeding leading to hospitalisation or death in patients with chronic atrial fibrillation treated with warfarin. Thromb Haemost 2005; 94: 537-43.

16. Wittkowsky A, Boccuzzi S, Wogen J, et al. Frequency of concurrent use of warfarin with potentially interacting drugs. Pharmacotherapy 2004; 24: 1668-74.

17. Toes M, Jones A, Prescott L. Drug interactions with paracetamol. Am J Ther 2005; 12: 56-66.

18. Hinz B, Cheremina O, Brune K. Acetaminophen (paracetamol) is a selective cyclooxygenase-2 inhibitor in man. FASEB J 2008; 22: 383-90.

19. Lucas R, Warner T, Vojnovic I, et al. Cellular mechanisms of acetaminophen: role of cyclo-oxygenase. FASEB $J$ 2005; 19: 635-7.

20. Amariles P, Giraldo N, Faus M. Interacciones medicamentosas: aproximación 
para establecer y evaluar su relevancia clínica. Med Clin 2007; 129: 27 - 35.

21. Shalansky S, Lynd L, Richardson K, et al. Risk of warfarin-related bleeding events and supratherapeutic international normalized ratios associated with complementary and alternative medicine: a longitudinal analysis. Pharmacotherapy 2007; 27: 1237-47.

22. Andrews F. Retroperitoneal haematoma after paracetamol increased anticoagulation. Emerg Med J 2002; 19: 84-5.

23. Fitzmaurice D, Murray J. Potentiation of the anticoagulant effect of warfarin. Postgrad Med J 1997; 73: 439-40.

24. Gebauer M, Nyfort-Hansen K, Henschke P, et al. Warfarin and acetaminophen interaction. Pharmacotherapy 2003; 23: 109-12.

25. Kotirum S, Chaiyakunapruk N, Jampachaisri K, et al. Utilization review of concomitant use of potentially interacting drugs in Thai patients using warfarin therapy. Pharmacoepidemiol Drug Saf 2007; 16: 216-22.

26. Kwan D, Bartle W, Walker $S$. The effects of acetaminophen on pharmacokinetics and pharmacodynamics of warfarin. J Clin Pharmacol 1999; 39: 68-75.

27. Mahé I, Bertrand N, Drouet L, et al. Paracetamol: a haemorrhagic risk factor in patients on warfarin. Br J Clin Pharmacol 2005; 59: 371-4.

28. Mahé I, Bertrand N, Drouet $\mathbf{L}$, et al. Interaction between paracetamol and warfarin in patients: a double-blind, placebo-controlled, randomized study. Haematologica 2006; 91: 1621-7.

29. Parra D, Beckey N, Stevens G. The effect of acetaminophen on the international normalized ratio in patients stabilized on warfarin therapy. Pharmacotherapy 2007; 27: 675-83.

30. Thijssen H, Soute B, Vervoort L, et al. Paracetamol (acetaminophen) warfarin interaction: NAPQI, the toxic metabolite of paracetamol, is an inhibitor of enzymes in the vitamin K cycle. Thromb Haemost 2004; 92: 797-802.

31. Lassen J, Kjeldsen J, Antonsen S, et al. Interpretation of serial measurements of international normalized ratio for prothrombin times in monitoring oral anticoagulant therapy. Clin Chem 1995; 41: $1171-6$.

32. Shek K, Chan L, Nutescu E. Warfarin acetaminophen drug interaction revisisted. Pharmacotherapy 1999; 19: 1153-8.

33. Whyte I, Buckley N, Reith D, et al. Acetaminophen causes an increased international normalized ratio by reducing functional factor VII. Therap Drug Monitor 2000; 22: 742-8.

34. Thijssen H, Soute B, Vervoort L, Claessens J. Paracetamol (acetaminophen) warfarin interaction: NAPQI, the toxic metabolite of paracetamol, is an inhibitor of enzymes in the vitamin K cycle. Thromb Haemost 2004; 92: 797-802. 